\title{
Morphological Characteristics of the Lacrimal Apparatus in its Obstruction of Various Genesis
}

\section{Grigory A. Demyashkin}

I M Sechenov First Moscow State Medical University: Pervyj Moskovskij gosudarstvennyj medicinskij universitet imeni I M Secenova

\section{Vasily D. Yartsev ( $D$ yartsew@ya.ru )}

Scientific Research Institute of Eye Diseases https://orcid.org/0000-0003-2990-8111

\section{Eugenia L. Atkova}

Scientific Research Institute of Eye Diseases

\section{Maxim A. Ekaterinchev}

Scientific Research Institute of Eye Diseases

\section{Vladimir I. Shchekin}

I M Sechenov First Moscow State Medical University: Pervyj Moskovskij gosudarstvennyj medicinskij universitet imeni I M Secenova

\section{Research Article}

Keywords: nasolacrimal duct, radioactive iodine, complication, pathomorphology

Posted Date: January 11th, 2022

DOI: https://doi.org/10.21203/rs.3.rs-1233320/v1

License: (c) (i) This work is licensed under a Creative Commons Attribution 4.0 International License.

Read Full License 


\section{Abstract}

Purpose: morphological assessment of the lacrimal ducts at various anatomical levels in patients with primary (PANDO) and secondary (SALDO) obstruction after radioactive iodine therapy.

Methods. The material was obtained during endoscopic dacryocystorinostomy with revision of Hasner's valve in patients with PANDO $(n=7)$ in the distal segments of the nasolacrimal duct and in patients with SALDO $(n=7)$ after radioactive iodine therapy. During the surgery, a biopsy of Hasner's valve, as well as a biopsy of the lacrimal sac wall were performed. The resulting material was stained with hemotoxylin and eosin, alcyan blue and by Masson method. Morphological and morphometric analyses were performed in semi-automatic mode. The results of histochemical staining of sections were translated into points taking into account the area and optical density (chromogenicity) in relative units: 1 - weak $(0-0.3) ; 2$ moderate $(0.3-0.6) ; 3$ - significant $(>0.6)$. The nonparametric Mann-Whitney criterion was used for statistical analysis. The differences were considered significant at $p<0.05$.

Results. The comparative morphological study both confirmed the available information concerning the radiation nature of the obstruction and allowed to quantify the fibrosis level of the stromal component and other lacrimal ducts structures.

Conclusion. It was shown that the nasolacrimal duct sclerosis is significantly lower $(p=0.029)$ in patients with SALDO than in patients with PANDO while fibrosis in the lacrimal sac is the same in patients of the compared groups.

\section{Background}

The cases of primary acquired nasolacrimal duct obstruction (PANDO) when the reason of its development is unclear are quite common in clinical practice. The cases when the factor that triggered nasolacrimal duct obstruction is known are less common. Such obstructions are called secondary acquired (SALDO). A special case of SALDO is obliteration resulting from radioactive iodine exposure during high-dose therapy for differentiated thyroid cancer described for the first time by R.T. Kloos[1].

Experience has shown that such obstruction develops most often in the distal segments of the nasolacrimal duct and is characterized by some pathomorphological features that distinguish primary and secondary acquired lacrimal drainage system obstruction[2]. A comparative study of changes in the lacrimal drainage system at various anatomical levels in patients with PANDO and SALDO due to radioactive iodine therapy has not yet been conducted, although the results of such a study would provide information concerning the pathogenesis of these conditions.

The purpose: morphological assessment of the lacrimal ducts at various anatomical levels in patients with primary (PANDO) and secondary (SALDO) obstruction after radioactive iodine therapy.

\section{Material And Methods}


The study was conducted after approval by the local Ethics Committee of Federal State Scientific Institution Research Institute of Eye Diseases. Informed voluntary consent of each patient for a biopsy and subsequent examination was obtained. In all cases, the biopsy was performed as part of the planned surgical intervention and did not affect the surgical approach and outcome of the surgery. The sampling was carried out at Federal State Scientific Institution Research Institute of Eye Diseases, laboratory studies were carried out on the basis of Sechenov University.

\section{Clinical and Demographic Data}

The material was obtained during endoscopic dacryocystorinostomy with revision of Hasner's valve in patients with PANDO $(n=7)$ in the distal segments of the nasolacrimal duct and in patients with SALDO $(n=7)$ after radioactive iodine therapy.

The average age of patients with PANDO was $57 \pm 18$ years ( $40-80$ years), the average age of patients with SALDO was $54 \pm 9$ years (44-56 years). The gender ratio of women and men was 6:1 in both groups. The average radiological activity administered in patients with SALDO was $114 \pm 17 \mathrm{mCi}(100-135 \mathrm{mCi})$, surgical intervention was performed $32 \pm 3$ months (29-36 months) after radioactive iodine therapy and $5 \pm 6$ months ( $0-13$ months) after complaints of lacrimation.

In all cases, patients complained of pronounced lacrimation rated 4 points on Munk scale, as well as the periodic mucopurulent lacrimal ducts discharge.

All patients underwent a conventional ophthalmological and dacryological examination, as well as CTdacryocystography to localize the nasolacrimal duct obstruction.

\section{Obtaining the Material}

After anemization of the nasal mucous membranes and infiltration anesthesia of the inferior nasal concha, it was displaced anteriorly. After eyeball instillation anesthesia, $n$. infraorbitalis conduction anesthesia and infiltration anesthesia, a probe with visual endoscopic position control was inserted antegradically into the lacrimal ducts of the inferior nasal meatus.

The probe displaced the soft tissues of Hasner's valve anteriorly. In area where the soft tissues protrusion was the greatest, an incision of Hasner's valve was made using a sickle-shaped scalpel, then a fragment of the nasolacrimal duct wall was removed with Blakesley forceps and sent to the pathohistological laboratory.

Then classical endoscopic dacryocystorhinostomy was performed. Following the opening of the lacrimal sac, protecting the formed flap of the lacrimal sac with the antegrade probe, a fragment of its posterior flap was removed with Blakesley forceps. This fragment was also sent to the pathohistological laboratory. 


\section{Morphological Study}

Fragments of the lacrimal sac and nasolacrimal duct obtained during the surgery were fixed in buffered formalin, processed in automatic mode (Leica Biosystems tissue histological processing apparatus, Germany), serial sections ( 2 microns thick) were classically stained with hematoxylin and eosin, as well as by Masson method and alcyan blue.

Morphological and morphometric analyses were performed in ten randomly selected fields of microscope at $\times 100$ and $\times 400$ magnification in four randomly selected sections of each sample, moving the slides at equal intervals along the $X$ and $Y$ axes using a semi-automatic image analyzer. Digital images of histological sections for morphometric studies were obtained using a video microscopy system (Leica DM3000 microscope, Leica DFC450 C camera, Germany), and morphometric data were obtained using Leica Application Suite (LAS) Version 4.9.0 (Germany) image processing and analysis software. To calculate the area of the stromal component, the images were automatically normalized and transferred from the 24-bit color image mode (RGB) to 256 gray scale mode, binarization of objects with a given sensitivity level was performed. When processing microphotographs by the image analysis system, binary objects with brightness $>150$ were cut off and the total area of positively colored objects of the total area of microphotographs was determined. The results of histochemical staining of sections were translated into points taking into account the area and optical density (chromogenicity) in relative units: 1 - weak (0 - 0.3); 2 - moderate $(0.3-0.6) ; 3$ - significant $(>0.6)$.

\section{Statistical Analysis}

The obtained data were processed using the IBM SPSS Statistics 26 computer program (IBM AnalyticS, USA). The resulting distribution of values was abnormal according to Shapiro-Wilk criterion. The nonparametric Mann-Whitney criterion was used for statistical analysis. The differences were considered significant at $p<0.05$.

\section{Study Outcome}

Signs of obliteration and punctate wall ulceration were observed in all samples of the lacrimal sac and nasolacrimal duct obtained from the 1st group patients (SALDO). A significant decrease in the height of the nasolacrimal duct single-layer cylindrical epithelium and autolytic changes in cytoplasm and microvacuolation and partial desquamation were noted (Fig. 1A, Fig. 2A). Secretory cells of the integumentary epithelium and mucous glands showed different staining on sulfated mucoid substances, hyaluronic acid and sialomucins which correlated with their morphofunctional state: starting with necrobiotic changes (significant basophilia of cells) and ending with various stages of dystrophic changes, including necrotic leading to lighter staining of cells with a disturbed structure (Fig. 1B, Fig. 2B). Most of the acinuses were necrotized, some of them had reduced lumens and abundant microvesicles in the cytoplasm; epithelial cells were hyperchromic. The cell nuclei were protruding, often pyknotic. In some 
cases, a reversible necrobiotic cells state which was replaced by necrotic changes in the most differentiated and vulnerable cells was noted: pale staining of the cytoplasm due to autolytic processes, as well as fuzzy intercellular boundaries, partial cellular desquamation associated with karyopycnosis and/or karyolysis were observed. A preserved oxyphilic basement membrane was found around the acinar shadows. Stromal component had moderate and severe fibrosis (Fig. 1C), as well as diffuse necrobiosis. Most of the small-caliber arteries were spasmed, the lumens of the arterioles were narrowed, and significant parts of the hemocapillaries were collapsed and emptied.

Histological examination of biopsies of the lacrimal sac and nasolacrimal duct obtained from the 2nd group patients (PANDO) revealed keratinization of the integumentary epithelium with squamous metaplasia, as well as epithelial cells with dystrophic changes (Fig. 2A). The cytoplasm of glandular cells was stained with mucins and non-sulfated glycosaminoglycans (Fig. 2B). Most acinuses were lined with epithelium with signs of atrophy due to the compression of glandulocytes by surrounding fibrous tissue, denudation and ischemic changes. Edema, a pronounced fibroplastic reaction with inflammatory infiltration and areas of fibrosis were observed in the stroma (Fig. 2C). At higher magnification it was found that the infiltrate consisted of lymphocytes and polymorphonuclear leukocytes which may indicate chronic active inflammation. Vascular congestion was observed.

Histochemical method - Masson's staining - for assessing the fiber component of the intercellular substance revealed a slight increase in collagen fiber bundles (blue staining) and elastic fibers bundles (red-yellow staining) in the lacrimal sacs in the second group patients (PANDO) compared with the first group (SALDO) representing 3.0 and 2.0 points, respectively (Fig. 1C, Fig. 3C).

At the same time, in nasolacrimal duct biopsies of the second group patients (PANDO), an increase in collagen fibers by 1.5 times ( 3.0 points) and a decrease in elastic fibers by 3.0 times ( 1.0 points) compared to the first group (SALDO) in which the collagen-elastic ratio was 2.0:3.0 were observed. (Fig. 2C, Fig. 4C).

The results of quantitative morphometric analysis of the fibrous component of the lacrimal sac and nasolacrimal duct biopsies in patients with SALDO and PANDO are presented in Table 1. When conducting pairwise comparisons, a statistically significant difference $(p=0.029)$ was found only when comparing the distribution of collagen and elastic fibers in biopsies obtained from Hasner's valve in patients with PANDO and SALDO. Significant differences were also observed when comparing the interaction between elastin and collagen in patients with PANDO and SALDO.

\section{Table 1}

Histochemical evaluation of collagen and elastic fibers proportion in patients with SALDO and PANDO, $\%$. 


\begin{tabular}{|lllllllll|}
\hline Value & \multicolumn{1}{l}{ Sac } & \multicolumn{9}{l|}{ Ductus } \\
& \cline { 2 - 10 } & \multicolumn{2}{l}{ Collagen, $\%$} & \multicolumn{2}{l|}{ Elastin, $\%$} & \multicolumn{2}{l|}{ Collagen, $\%$} & \multicolumn{2}{l|}{ Elastin, \% } \\
\cline { 2 - 10 } & PANDO & SALDO & PANDO & SALDO & PANDO & SALDO & PANDO & SALDO \\
\hline Mean & 54.6 & 53.1 & 10.1 & 10 & $\mathbf{5 0 . 9}$ & $\mathbf{3 2}$ & $\mathbf{6 . 1}$ & $\mathbf{1 8 . 3}$ \\
\hline St. deviation & 19.7 & 19 & 5.9 & 5.5 & 14.3 & 12 & 3.2 & 13.1 \\
\hline
\end{tabular}

\section{Discussion}

According to the study, SALDO occurs in $8.8 \%$ of patients after a single high-dose radioactive iodine therapy, and the probability of the complication is multiplied during repeated treatment[3]. The analysis of the lacrimal sac and nasolacrimal duct biopsies revealed the expression of $\mathrm{Na}^{+} / /$'symporter (NIS) in these structures which may be the biochemical basis for the development of SALDO[4]. The modern theory of SALDO pathogenesis due to exposure to radioactive iodine considers this complication a consequence of radioactive iodine in the tear fluid and its active capture by the tear ducts through the opening of NIS protein channel [5-7]. Imaging studies have confirmed that during radioactive iodine therapy fixation of a radioactive drug by nasal cavity and lacrimal ducts is possible[5, 6]. Observations show that SALDO most often develops in the distal parts of the nasolacrimal duct, in particular, at the level of Hasner's valve, although there are cases of more proximal obstruction, including at the level of the lacrimal sac which is accompanied by dacryocystitis.

Previous studies on the comparative pathomorphological analysis of changes in Hasner's valve in PANDO and SALDO due to radioactive iodine therapy have shown that in the latter case specific changes characterized as radiation damage to Hasner's valve develop[2]. It was found that in SALDO desquamation of the nasolacrimal duct epithelium and damage to the mucous glands are observed which is accompanied by moderate fibrosis, while in PANDO fibrosis is relatively more pronounced and is apparently a consequence of a long-running chronic inflammatory process.

We have proposed the hypothesis that the pathological process, primarily induced in the distal segments of the nasolacrimal duct, leads to the obstruction of the lacrimal ducts in situ, and proximal obstruction is a consequence of secondary alteration resulting from excessive reactivity of the tissue. This hypothesis required pathomorphological verification consisting in a comparative study of changes in the lacrimal ducts at various anatomical levels, as well as in the dependence on cause of the obstruction. Of particular interest is the study of the fibrous component of the lacrimal duct wall, since it was previously shown that it is sclerotic changes that result in SALDO. Such a study has been achieved by Masson's trichrome staining, as well as through the use, in addition to the classical, digital analysis, of the images with a quantitative histochemical evaluation of the results. The alcyan blue staining enabled to characterize the changes in the mucous glands the role of which in the development of SALDO was explained previously. 
The study confirmed the results of previous study on the relative differences in pathomorphological changes in Hasner's valve in SALDO and PANDO. In addition, statistically significant $(p=0.029)$ differences in the ratio of collagen and elastin fibers in Hasner's valve in patients with SALDO and PANDO were found which is apparently a consequence of the lower severity of fibrotic processes in patients with SALDO after radioactive iodine exposure than in patients with PANDO. At the same time, similar ratios in the structure of lacrimal sacs, although they were identified, were recognized as statistically unreliable which enables to characterize the severity of fibrosis in the lacrimal sac as the same in SALDO due to radioactive iodine therapy and in PANDO. The above confirms our hypothesis according to which the primary nasolacrimal duct obstruction after radioactive iodine treatment occurs in the distal parts of the nasolacrimal duct, and the inducing inflammation and fibrotization in the more proximal parts of the lacrimal ducts are secondary. Indirect evidence of these assumptions is the analysis of the anatomical structure of SALDO due to radioactive iodine therapy in the clinical cases described by other authors which, despite the known limitations of such analysis associated with statistically unreliable samples and inaccurate case description, shows the relative predominance of distal forms of such obstruction[5, 7-13].

\section{Conclusion}

Thus, the comparative morphological study of biopsies of the lacrimal sac and Hasner's valve in patients with SALDO in radioactive iodine treatment and in patients with PANDO not only confirmed the available information regarding the radiation nature of the lesion, but also enabled to quantify the level of fibrosis of the stromal component and other lacrimal ducts structures. It was shown that the nasolacrimal duct sclerosis is significantly lower $(\mathrm{p}=0.029)$ in patients with SALDO than in patients with PANDO, while fibrosis in the lacrimal sac is the same in patients of the compared groups. This circumstance confirms the hypothesis that the primary lesion when exposed to radioactive iodine affects Hasner's valve, and the alteration of proximal structures is secondary and similar in its pathomorphological characteristics to the process developing with PANDO.

\section{Declarations}

\section{Funding.}

This work was supported by Scientific Research Institute of Eye Diseases.

\section{Compliance with ethical standard.}

This work complies with the ethical standards of the institutional and with the 1964 Helsinki Declaration and its later amendments.

\section{Conflict of interest.}


The authors declared no potential conficts of interest with respect to the research, authorship, and/or publication of this article.

\section{Ethical approval.}

This work has received the ethical approval from Biomedical Ethics Committee, Scientific Research Institute of Eye Diseases.

\section{Informed consent.}

All participants of this study have given their written informed consent for the research and for publication.

\section{Authorship.}

All authors meet all criteria for authorship according to ICMJE.

\section{References}

1. Kloos RT, Duvuuri V, Jhiang SM, Cahill KV, Foster JA, Burns JA (2002) Nasolacrimal drainage system obstruction from radioactive iodine therapy for thyroid carcinoma. J Clin Endocrinol Metab 87:58175820. DOI 10.1210/jc.2002-020210

2. Fedorov AA, Atkova EL, Yartsev VD (2020) Secondary Acquired Nasolacrimal Duct Obstruction as a Specific Complication of Treatment With Radioactive lodine (Morphological Study). Ophthalmic Plast Reconstr Surg 36:250-253. DOI 10.1097/IOP.0000000000001521

3. Yartsev VD, Solodkiy VA, Fomin DK, Borisenko TE, Atkova EL (2021) Clinical and Demographic Characteristics of Tearing in Patients after Radioiodine Ablation for Differentiated Thyroid Cancer. Curr Eye Res 46:1320-1324. DOI 10.1080/02713683.2021.1878229

4. Morgenstern KE, Vadysirisack DD, Zhang Z, Cahill KV, Foster JA, Burns JA, Kloos RT, Jhiang SM (2005) Expression of sodium iodide symporter in the lacrimal drainage system: implication for the mechanism underlying nasolacrimal duct obstruction in I(131)-treated patients. Ophthal Plast Reconstr Surg 21:337-344

5. Ali MJ (2016) lodine-131 Therapy and Nasolacrimal Duct Obstructions: What We Know and What We Need to Know. Ophthal Plast Reconstr Surg 32:243-248. DOI 10.1097/IOP.0000000000000647

6. Ali MJ, Vyakaranam AR, Rao JE, Prasad G, Reddy PV (2017) lodine-131 Therapy and Lacrimal Drainage System Toxicity: Nasal Localization Studies Using Whole Body Nuclear Scintigraphy and SPECT-CT. Ophthal Plast Reconstr Surg 33:13-16. DOI 10.1097/IOP.0000000000000603 
7. Al-Qahtani KH, Al Asiri M, Tunio MA, Aljohani NJ, Bayoumi Y, Munir I, AlAyoubi A (2014) Nasolacrimal duct obstruction following radioactive iodine 131 therapy in differentiated thyroid cancers: review of 19 cases. Clin Ophthalmol 8:2479-2484. DOI 10.2147/OPTH.S71708

8. da Fonseca FL, Yamanaka PK, Kato JM, Matayoshi S (2016) Lacrimal System Obstruction After Radioiodine Therapy in Differentiated Thyroid Carcinomas: A Prospective Comparative Study. Thyroid 26:1761-1767. DOI 10.1089/thy.2015.0657

9. Brockmann H, Wilhelm K, Joe A, Palmedo H, Biersack HJ (2005) Nasolacrimal drainage obstruction after radioiodine therapy: case report and a review of the literature. Clin Nucl Med 30:543-545

10. Burns JA, Morgenstern KE, Cahill KV, Foster JA, Jhiang SM, Kloos RT (2004) Nasolacrimal obstruction secondary to I(131) therapy. Ophthal Plast Reconstr Surg 20:126-129

11. Fard-Esfahani A, Farzanefar S, Fallahi B, Beiki D, Saghari M, Emami-Ardekani A, Majdi M, Eftekhari M (2012) Nasolacrimal duct obstruction as a complication of iodine-131 therapy in patients with thyroid cancer. Nucl Med Commun 33:1077-1080. DOI 10.1097/MNM.0b013e3283570fb8

12. Lee SL (2010) Complications of radioactive iodine treatment of thyroid carcinoma. J Natl Compr Canc Netw 8:1277-1286. quiz 1287

13. Yuoness S, Rachinsky I, Driedger AA, Belhocine TZ (2011) Differentiated thyroid cancer with epiphora: detection of nasolacrimal duct obstruction on I-131 SPECT/CT. Clin Nucl Med 36:11491152. DOI 10.1097/RLU.0b013e3182336016

\section{Figures}

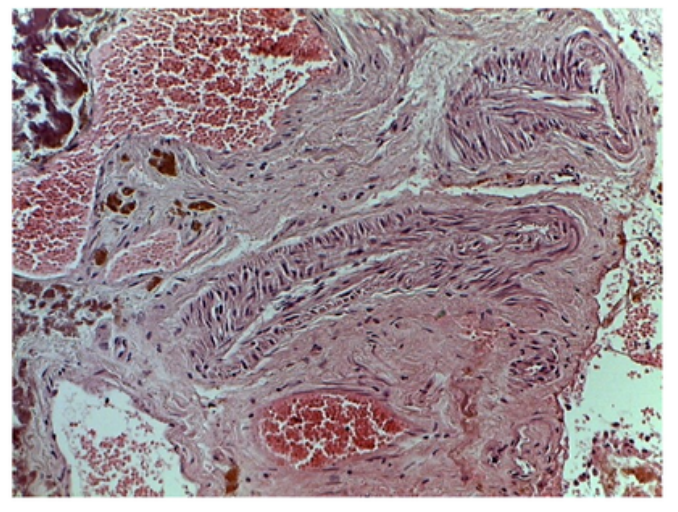

a

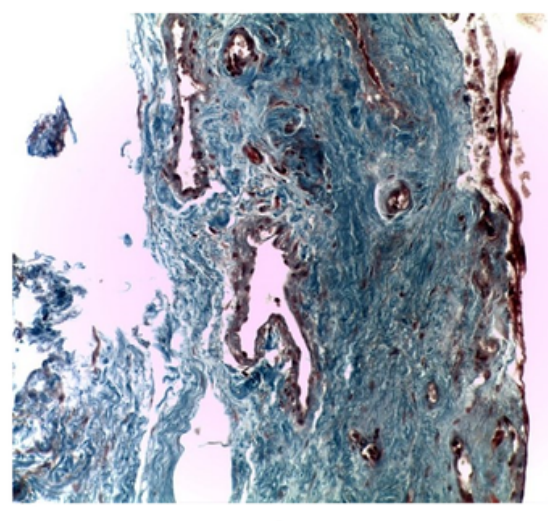

b

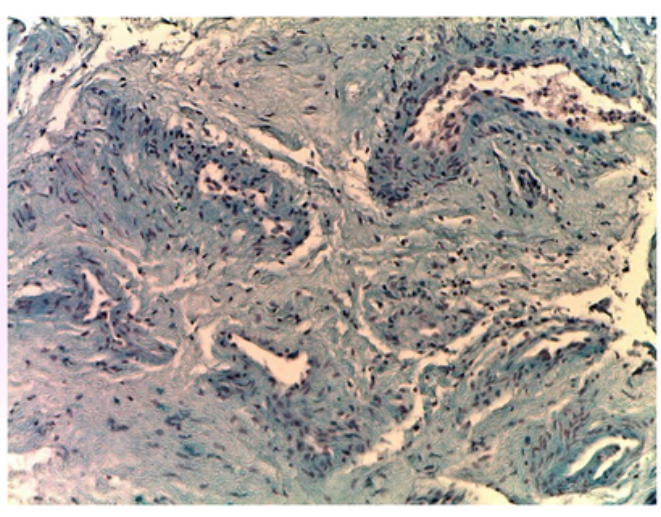

c

\section{Figure 1}

Lacrimal sac in SALDO group, magnified $\times 200$. Staining: A - hematoxylin and eosin; B - alcyan blue; C Masson Trichrome 


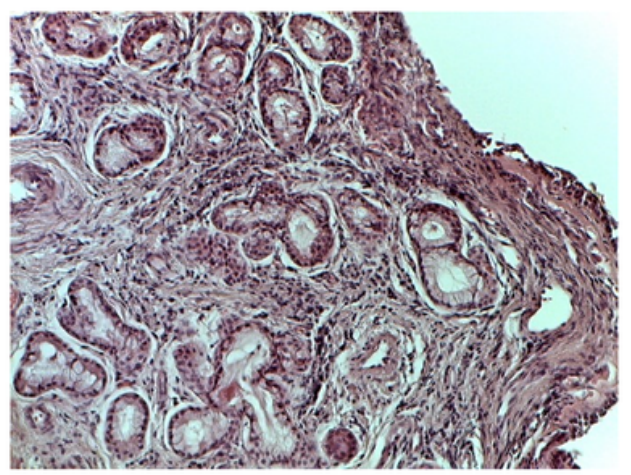

a

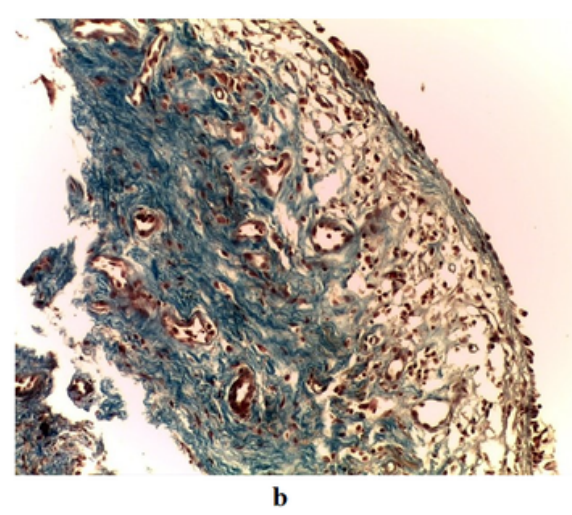

b

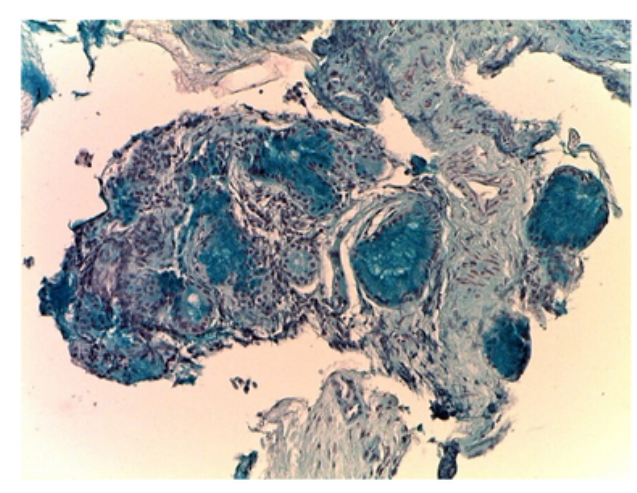

c

\section{Figure 2}

Nasolacrimal duct in SALDO group, magnified $\times 200$. Staining: A - hematoxylin and eosin; B - alcyan blue; C - Masson Trichrome

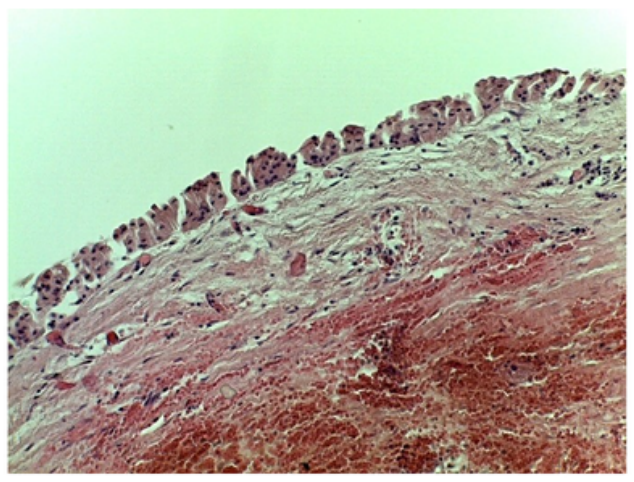

a

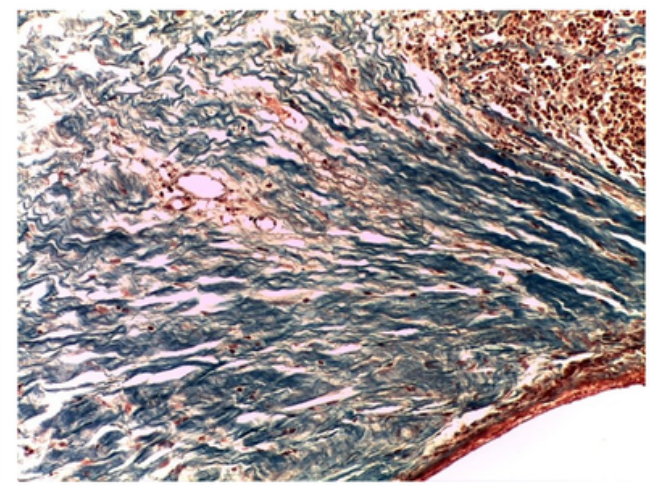

b

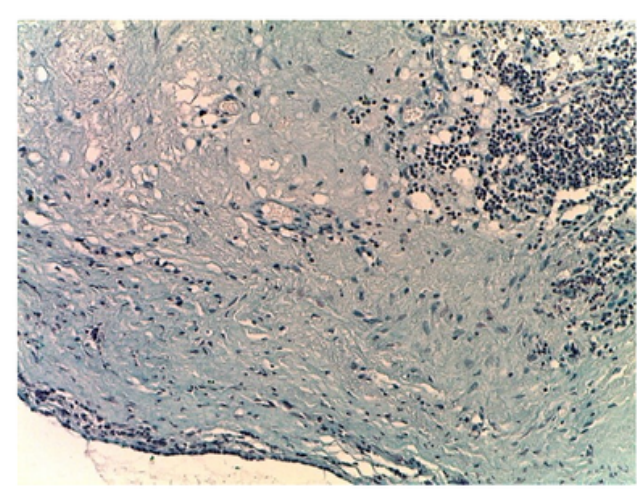

c

\section{Figure 3}

Lacrimal sac in PANDO group, magnified $\times 200$. Staining: A - hematoxylin and eosin; B - alcyan blue; C Masson Trichrome 


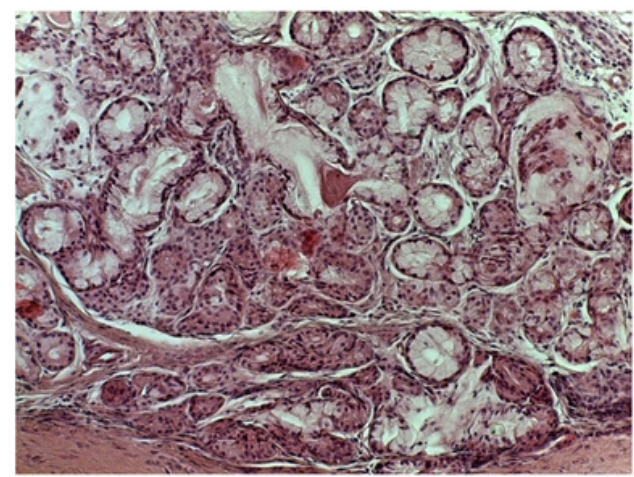

a

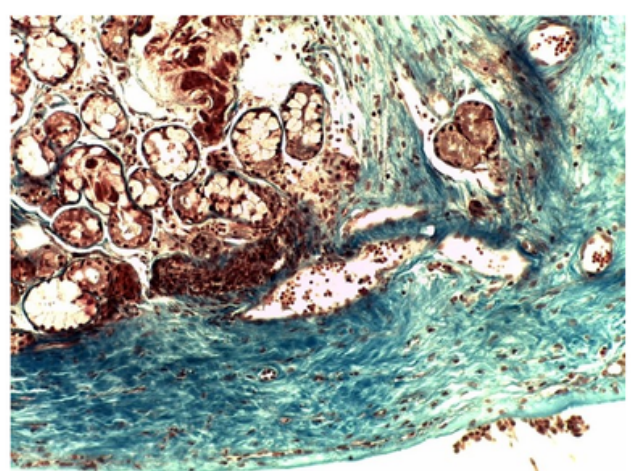

b

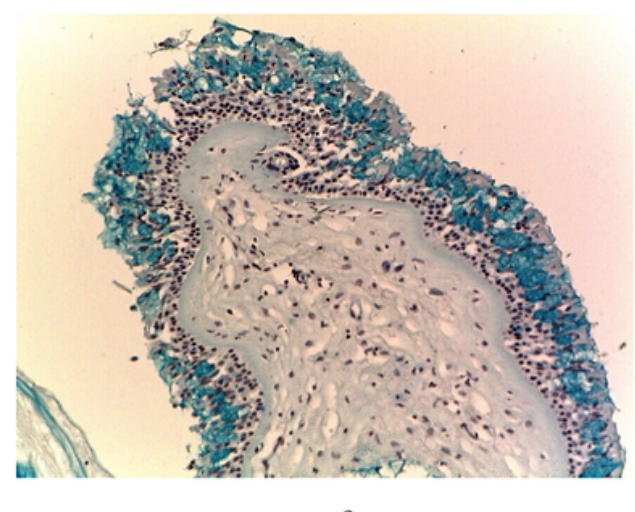

c

\section{Figure 4}

Nasolacrimal duct in PANDO group, magnified $\times 200$. Staining: A - hematoxylin and eosin; B - alcyan blue; C - Masson Trichrome 